OPEN ACCESS

Edited by:

Xinchun Jin,

Capital Medical University, China

Reviewed by:

Peiying Li,

Shanghai Jiao Tong University School of Medicine, China

Xiaohuan Xia,

Tongji University, China

*Correspondence:

Han Liu

fccliuh2@zzu.edu.cn

Huangui Xiong

hxiong@unmc.edu

Yuming $X u$

xuyuming@zzu.edu.cn

${ }^{\dagger}$ These authors have contributed equally to this work and share first authorship

Specialty section:

This article was submitted to

Non-Neuronal Cells,

a section of the journal

Frontiers in Cellular Neuroscience

Received: 11 March 2021

Accepted: 20 May 2021

Published: 22 June 2021

Citation:

Liu $H$, Yang $X$, Yang J, Yuan $Y$, Wang $Y$, Zhang $R$, Xiong $H$ and $X u Y$ (2021) IL-17 Inhibits Oligodendrocyte

Progenitor Cell Proliferation and Differentiation by Increasing $\mathrm{K}^{+}$

Channel Kv1.3.

Front. Cell. Neurosci. 15:679413.

doi: 10.3389/fncel.2021.679413

\section{IL-17 Inhibits Oligodendrocyte Progenitor Cell Proliferation and Differentiation by Increasing $\mathrm{K}^{+}$ Channel Kv1.3}

Han Liu' ${ }^{1 * t}$, Xueke Yang ${ }^{1 \dagger}$, Jing Yang1, Yanpeng Yuan ${ }^{1}$, Yanlin Wang ${ }^{1}$, Rui Zhang ${ }^{1}$, Huangui Xiong ${ }^{2 *}$ and Yuming $\mathrm{Xu}^{1 *}$

\begin{abstract}
' Department of Neurology, The First Affiliated Hospital of Zhengzhou University, Zhengzhou, China, ${ }^{2}$ Neurophysiology Laboratory, Department of Pharmacology and Experimental Neuroscience, University of Nebraska Medical Center, Omaha, NE, United States
\end{abstract}

Interleukin 17 (IL-17) is a signature cytokine of Th17 cells. IL-17 level is significantly increased in inflammatory conditions of the CNS, including but not limited to post-stroke and multiple sclerosis. IL-17 has been detected direct toxicity on oligodendrocyte (OI) lineage cells and inhibition on oligodendrocyte progenitor cell (OPC) differentiation, and thus promotes myelin damage. The cellular mechanism of IL-17 in CNS inflammatory diseases remains obscure. Voltage-gated $\mathrm{K}^{+}(\mathrm{Kv})$ channel 1.3 is the predominant $\mathrm{Kv}$ channel in $\mathrm{Ol}$ and potentially involved in $\mathrm{Ol}$ function and cell cycle regulation. Kv1.3 of $\mathrm{T}$ cells involves in immunomodulation of inflammatory progression, but the role of Ol Kv1.3 in inflammation-related pathogenesis has not been fully investigated. We hypothesized that IL-17 induces myelin injury through Kv1.3 activation. To test the hypothesis, we studied the involvement of OPC/OI Kv1.3 in IL-17-induced OI/myelin injury in vitro and in vivo. Kv1.3 currents and channel expression gradually decreased during the OPC development. Application of IL-17 to OPC culture increased Kv1.3 expression, leading to a decrease of AKT activation, inhibition of proliferation and myelin basic protein reduction, which were prevented by a specific Kv1.3 blocker 5(4-phenoxybutoxy) psoralen. IL-17-caused myelin injury was validated in LPC-induced demyelination mouse model, particularly in corpus callosum, which was also mitigated by aforementioned Kv1.3 antagonist. IL-17 altered Kv1.3 expression and resultant inhibitory effects on OPC proliferation and differentiation may by interrupting AKT phosphorylating activation. Taken together, our results suggested that IL-17 impairs remyelination and promotes myelin damage by Kv1.3-mediated OI/myelin injury. Thus, blockade of Kv1.3 as a potential therapeutic strategy for inflammatory CNS disease may partially attribute to the direct protection on OPC proliferation and differentiation other than immunomodulation.

Keywords: IL-17, myelin, oligodendrocyte, Kv1.3, inflammation 


\section{INTRODUCTION}

In the central nervous system (CNS), neuronal axons are myelinated with oligodendrocytes (Ols), and damage of such a myelin sheath is a striking pathological feature of white matter damage in many inflammation-related diseases, including stroke (Oksala et al., 2009), Alzheimer disease (Nasrabady et al., 2018), and in particular multiple sclerosis (MS), which is a disseminated sclerosis in CNS affecting millions of people worldwide. While the mechanisms underlying MS pathogenesis are not fully understood, it is widely accepted that myelin sheath destruction induced by autoimmune response is most likely the cause. In MS, the immune system attacks myelin sheath leading to demyelination and impairs remyelination by retardation or inhibition of the myelin-producing cells to form myelin sheath after demyelination (Boulanger and Messier, 2014). Remyelination requires Ol progenitor cell (OPC) proliferation and migration to the lesion sites, where they differentiate, eventually to mature Ols and wrap neuronal axons to form myelin sheath. Remyelination readily occurs in MS, but it is incomplete and inefficient, and the reason is unfortunately unknown. It is believed that OPC differentiation is the key step for successful remyelination based on the fact revealed by pathological studies reported that approximately 60 $70 \%$ of demyelinated lesions in MS contain immature OPCs. Those immature OPCs appeared to be in an arrested state, unable to fully differentiate (Lucchinetti et al., 1999; Chang et al., 2002; Boyd et al., 2013). Although current therapies targeting at suppression of overactive immune cells, such as lymphocytes, have some promising effects in retarding disease progression (Hauser et al., 2013), there is no effective way to stimulate and promote axonal remyelination once a demyelinated lesion has occurred.

The Voltage-gated $\mathrm{K}+(\mathrm{Kv})$ channel is a largest and rapidly growing family of ion channels. In general, $\mathrm{Kv}$ channels are responsible for the driving force of $\mathrm{Ca}^{2+}$ in non-excitable cells, thus mediating a variety of cellular activities. Previous studies have revealed an involvement of $\mathrm{Kv}$ channels in the regulation of $\mathrm{Ol}$ lineage cell proliferation and maturation (Pruss et al., 2011). It has also been shown that a decrease in Kv1.3 and Kv1.5 channel expression and outward $\mathrm{K}^{+}$currents in mature Ols is essential for synthesis of myelin structural proteins, and suppression of outward $\mathrm{K}^{+}$currents by increasing extracellular $\mathrm{K}^{+}$concentration (e.g., $25 \mathrm{mM} \mathrm{KCl}$ ) promotes $\mathrm{Ol}$ maturation (Dai et al., 2001). These results indicate that Kv channels play an important role in the regulation of $\mathrm{OPC} / \mathrm{Ol}$ differentiation and maturation (Chittajallu et al., 2002; Tegla et al., 2011). Kv1.3 is highly elevated in memory T cells in MS brain (Rus et al., 2005) and has been reported to be an immune-regulatory target in MS and inflammatory animal models (Wang et al., 2019). The inhibition of Kv1.3 activity may attenuate CNS inflammation and thus benefit remyelination according to its crucial role in regulation of $\mathrm{T}$ lymphocytes (Rus et al., 2005; Schmitz et al., 2005; Eil et al., 2016; Wang et al., 2019) and microglial physiological activity (Fordyce et al., 2005; Di Lucente et al., 2018) in inflammatory brains. Thus, blockade of Kv1.3 might benefit CNS remyelination by suppression of immune response. However, Kv1.3 colocalized with OPC marker NG2 in MS brain (Tegla et al., 2011), which suggests the beneficial effects of Kv1.3 blockade on MS may not be attributable solely to immune suppression; direct protection on OPCs may also contribute to the observed outcome. Nevertheless, the role of OPC/Ol Kv1.3 on axonal remyelination in brain white matter damage is unknown. In a previous study, we showed Kv1.3 was involved in HIV Tat protein-induced $\mathrm{Ol}$ injury in rat corpus callosum (Liu et al., 2017a). To further evaluate the role of $\mathrm{OPC} / \mathrm{Ol} \mathrm{Kv1.3} \mathrm{on} \mathrm{remyelination} \mathrm{in} \mathrm{white} \mathrm{matter} \mathrm{damage,} \mathrm{we}$ studied the effects of interleukin 17 (IL-17) on regulation of $\mathrm{OPC} / \mathrm{Ol}$ proliferation and differentiation via Kv1.3.

IL-17 is a member of cytokine family of IL-17A-F (Waisman et al., 2015). As a signature cytokine of Th17 cells, IL-17A (referred to as IL-17 hereafter) is significantly elevated in MS patients' cerebrospinal fluid (CSF) (Kostic et al., 2014) and induces demyelination ( $\mathrm{Li}$ et al., 2013; Waisman et al., 2015; Wang et al., 2017), due to its proinflammatory nature and direct toxicity on OPCs/Ols, as well as strong inhibitory effects on OPC maturation (Paintlia et al., 2011; Kang et al., 2013). Numerous immune-regulatory functions have been reported for IL-17 (Waisman et al., 2015; Kolbinger et al., 2016; Moser et al., 2020). It stimulates immune cell production of proinflammatory molecules leading to neurodegeneration, and its elevated levels in the CSF are associated with CNS inflammatory diseases. In addition, Kv1.3 expression has been found to correspond with an increase in IL-17 secretion in T cells (Gocke et al., 2012; Koch Hansen et al., 2014; Grishkan et al., 2015). To this end, we used IL17 as a proinflammatory agent to investigate the inflammationrelated mechanisms in OPC proliferation and differentiation through regulation of the levels of Kv1.3 expression.

\section{MATERIALS AND METHODS}

\section{Animals}

C57BL/6 male mice (21 days) were supplied by Charles River Laboratories, Beijing, China, and were housed in groups of five at the standard conditions of $22^{\circ} \mathrm{C} \pm 1{ }^{\circ} \mathrm{C}$ temperature and relative humidity conditions of $55-60 \%$ in an artificially lit animal room under a 12-h period of light and dark cycle and fed water and food ad libitum. Only male mice were used in the present research. This study was approved by the Life Science Ethics Review Committee of Zhengzhou University.

\section{Human OI Precursor Cell Culture and Differentiation}

Human Ol precursor cell was introduced from ScienCell Research Laboratories (San Diego, CA). Cells were cultured in complete medium containing 89\% RPMI 1640 medium (HyClone, Logan, UT) and supplemented with 10\% fetal bovine serum (Biological Industries, Beit-Haemek, Israel), 100 $\mathrm{U} / \mathrm{mL}$ penicillin, and $100 \mu \mathrm{g} / \mathrm{mL}$ streptomycin (P/S; Solarbio, Beijing, China). Cells were kept in an incubator at $37^{\circ} \mathrm{C}$ under a humidified atmosphere of $5 \% \mathrm{CO}_{2}$ and $95 \%$ air. OPCs were maintained in complete medium for $24 \mathrm{~h}$ to adhere to the flask, after which the medium was replaced by 
Ol precursor cell differentiation medium (OPCDM; ScienCell Research Laboratories, San Diego, CA) to differentiate into mature Ols. The experiments using Ols were performed with cells after 6 days or otherwise indicated cultured in OPCDM. OPCDM was replaced on a daily basis.

\section{Demyelination Mouse Model (Two-Point Injection)}

Mice were anesthetized with isoflurane (induced at 3\%, and maintained at $1.2-1.6 \%)$ and positioned in a stereotaxic frame. Corpus callosum demyelination was induced by stereotaxic injection of $2 \mu \mathrm{L}$ ( $1 \mu \mathrm{L}$ for each point) of $1 \%$ lysophosphatidylcholine (LPC; Sigma, St. Louis, MO), which was an endogenous lysophospholipid that disrupts myelin-associated lipids leading to focal demyelination, in $0.9 \% \mathrm{NaCl}$ solution at the rate of $0.5 \mu \mathrm{L} / \mathrm{min}$ using $1 \mu \mathrm{L}$ microsyringe at two points of corpus callosum. Mice of sham group were injected with equal volume of saline in double-point injection: (1) left: $1.0 \mathrm{~mm}$ lateral to the bregma, $1.1 \mathrm{~mm}$ anterior, and $2.4 \mathrm{~mm}$ deep; (2) right: $1.0 \mathrm{~mm}$ lateral to the bregma, $0.6 \mathrm{~mm}$ anterior, and $2.1 \mathrm{~mm}$ deep. After injection, the needle was kept in the place for an additional 5 min to prevent backflow. The day of injection was regarded as day 0 (0 dpi). For all groups, the mice were daily administered 5-bromo-2'-deoxyuridine (BrdU, $50 \mathrm{mg} / \mathrm{kg}$; Solarbio, Beijing, China) at 2, 3, and $4 \mathrm{dpi}$ by intraperitoneal (i.p.) injection. A specific Kv1.3 blocker 5-(4-phenoxybutoxy) psoralen (PAP, $6 \mathrm{mg} / \mathrm{kg}$; Santa Cruz Biotechnology, Santa Cruz, CA) was applied by i.p. injection for the PAP and PAP + LPC groups at 2, 3, and $4 \mathrm{dpi}$. The brain tissues were taken at $5 \mathrm{dpi}$ for cryostat section or Western blot analysis.

\section{Electrophysiology}

Isolation of Kv1.3 currents was achieved as previously described (Liu et al., 2017a). Briefly, cells were seeded onto 3.5-cm culture dishes for whole-cell voltage clamp recording. Cells were perfused with oxygenated standard bath solution contained (in $\mathrm{mM}$ ): $140 \mathrm{NaCl}, 5.4 \mathrm{KCl}, 2 \mathrm{CaCl}, 1 \mathrm{MgCl}_{2}, 10 \mathrm{HEPES} / \mathrm{NaOH}$, $\mathrm{pH}$ 7.3. The osmolarity was adjusted to $305 \mathrm{mOsm}$ prior to recording by $\mathrm{D}$-sucrose. The electrodes solution contained (in $\mathrm{mM}$ ): $140 \mathrm{KCl}, 2 \mathrm{CaCl} 2,2 \mathrm{MgCl}_{2}, 11 \mathrm{EGTA}, 10 \mathrm{HEPES} / \mathrm{KOH}$, $\mathrm{pH} 7.3$, and had an osmolarity of $300 \mathrm{mOsm}$. Whole-cell $\mathrm{K}^{+}$currents were evoked by applying voltage steps from -150 to $+60 \mathrm{mV}$ with a $15 \mathrm{mV}$ increments, and current amplitudes were measured at the peak for each test potential. Current density $(\mathrm{pA} / \mathrm{pF})$ was calculated by dividing the digitized current values by whole-cell capacitance, which represents cell membrane surface area. Stock solution of Kv1.3-specific inhibitor PAP (Santa Cruz Biotechnology, Santa Cruz, CA) was prepared in deionized water. To access Kv1.3 current isolation, cells were recorded in standard bath and then superfused with PAP-contained bath solution at a concentration of 10 nM. Kv1.3-excluded currents were recorded at $20 \mathrm{~min}$ after perfusion. Isolated Kv1.3 currents were obtained by subtraction of Kv1.3-excluded current from total outward $\mathrm{K}^{+}$currents. All experiments were done at room temperature $\left(22-23^{\circ} \mathrm{C}\right)$. Recordings were obtained with an Axopatch-200 B amplifier
(Molecular Devices, Sunnyvale, CA). Current signals were filtered at $1 \mathrm{kHz}$ and digitized at $5 \mathrm{kHz}$ using a Digidata 1440A interface (Molecular Devices). The current and voltage traces were displayed and recorded on a computer using pCLAMP 10.0 data acquisition and analysis software (pClamp, RRID:SCR_011323). Data were analyzed by Clampfit 10.0 (Clampfit, RRID:SCR_011323). All final graphics in the present work were constructed by GraphPad Prism 9.0 (GraphPad Prism, RRID:SCR_002798).

\section{3-(4,5-Dimethylthiazol-2-yl)-2,5-Diphenyl Tetrazolium Bromide Assay}

Cell proliferation was measured by 3-(4,5-dimethylthiazol2-yl)-2,5-diphenyl tetrazolium bromide (MTT) assay. The homogeneous stable solution of $5 \mathrm{mg} / \mathrm{mL}$ was prepared by dissolving MTT (Ameresco, Solon, $\mathrm{OH}$ ) powder in phosphatebuffered saline (PBS). Cells were seeded in a 96-well plate with complete medium. IL-17 (Absin Bioscience Inc., Shanghai, China) and AKT activator SC79 (Selleck Chemicals, Houston, TX) were added to the cell cultures for $48 \mathrm{~h}$ in the absence or presence of prior (30 min) added Kv1.3 antagonist PAP. Cells were then incubated with a 1:10 dilution of the MTT solution to complete medium for $3 \mathrm{~h}$ at $37^{\circ} \mathrm{C}$. The formazan crystals converted from MTT were completely dissolved in dimethyl sulfoxide (Solarbio) for cell lysis, and the optical density (OD) was measured at $570 \mathrm{~nm}$ using Multiskan Spectrum (Thermo Fisher Scientific, Waltham, MA). The ratio of OD between the treated cells and the control cells reflected cell viability.

\section{Immunocytochemistry}

OPCs were seeded on coverslips at a density of $0.02 \times 10^{6} /$ well in 24-well plates. Experimental treatments of PAP, IL-17, and SC79 were the same as described in MTT assay. After treatments as at indicated time, the prepared cells were fixed with 4\% paraformaldehyde (PFA) in PBS for $20 \mathrm{~min}$ and punched holes in PBST supplemented with $0.1 \%$ Triton-X100 for $20 \mathrm{~min}$. Cells were blocked in PBST containing 1\% bovine serum albumin (BSA; Solarbio) for $30 \mathrm{~min}$, and all the above experiments were performed at room temperature. Primary antibodies anti-Kv1.3 (Thermo Fisher Scientific cat. no. PA5-77618, RRID:AB_2736055) and anti-BrdU (ABclonal, cat. no.A20304, RRID:AB_2890022) were then applied to coverslips at $4^{\circ} \mathrm{C}$ overnight, and cells were incubated with the appropriate fluorescence-conjugated secondary antibody for $1 \mathrm{~h}$ at room temperature. For BrdU staining, cells on coverslips were pretreated with $\mathrm{HCl}$. After washing, the coverslips were mounted in glass slides with mounting medium contained DAPI stain, and cells were visualized by a fluorescent microscope (Nikon Corporation, Tokyo, Japan). The ratio of $\mathrm{BrdU}^{+}$cells to DAPI was counted, and comparisons among groups were performed.

\section{Immunohistochemistry}

The mice injected with LPC accepted BrdU by i.p. injection at 2, 3, and $4 \mathrm{dpi}$. The mice used for cryosectioning accepted cardiac perfusion before sacrifice. After anesthesia, the mice 
were fixed on the operating table, and the thoracic cavity was cut to expose the heart. The left ventricle was punctured with a syringe filled with precooled saline. Then, the right auricula dextra of mice were snipped, and the syringe was pushed to allow the saline to rinse systemically through the vessels. After the effluent turned limpid, the mice were perfused with $4 \%$ PFA to systemic circulation for prefixation. Each mouse was given $200 \mathrm{~mL}$ saline and $200 \mathrm{~mL}$ PFA. The prepared brain tissue specimens were fixed in 4\% PFA for $48 \mathrm{~h}$, immersing in $15 \%$ and $30 \%$ sucrose for $24 \mathrm{~h}$, respectively, and then embedded in optimal cutting temperature (OCT) media, freezing, and cryosectioning into $10-\mu \mathrm{m}$ slices. The coronal brain slices were immunostained with primary antibody such as anti-myelin basic protein (MBP) antibody (Abcam cat. no. ab7349, RRID:AB_305869), anti-NG2 monoclonal antibody (Thermo Fisher Scientific cat. no. 372300, RRID:AB_2533306), and anti-BrdU antibody (ABclonal, cat. no.A20304, RRID:AB_2890022) to evaluate demyelination and proliferation, respectively. The sections were incubated with primary antibody followed by fluorescence-conjugated secondary antibodies for microscopic analysis. A minimum of five images were taken from each slide.

\section{Luxol Fast Blue Stain}

Luxol fast blue (LFB) staining was used to measure the demyelination of myelin. The coronal brain slices were prepared as previously described. LFB staining was performed according to manufacturer's instructions for the Luxol fast blue stain kit (Solarbio). The slices were immerged in LFB dye at room temperature for $12 \mathrm{~h}$. The slices were rinsed with distilled water after washing off the excess dye with $95 \%$ ethanol. The slices were differentiated successively in $0.05 \%$ lithium carbonate solution and $70 \%$ ethanol. Photographs were taken with an optical microscope after sealing the slices with neutral resin.

\section{Western Blot Analysis}

The expression levels of proteins were quantified by Western blot. MBP has three isoforms, and we examined the predominant isoform of protein band at $18.5 \mathrm{kDa}$ by Western blot analysis. Following experimental treatments, cells and brain tissue were washed thrice with prechilled PBS. The whole-cell lysates were prepared in RIPA lysis buffer (Absin Bioscience Inc.) followed by clarification with centrifugation. All protein concentrations were quantified using the BCA protein assay kit (Solarbio), and $25 \mu \mathrm{g}$ of total protein was loaded onto $10 \%$ sodium dodecyl sulfate-polyacrylamide gels, separated by electrophoresis, and transferred to a polyvinylidene difluoride (PVDF; Millipore, Bedford, MA) membrane. The PVDF membrane was then blocked in 5\% non-fat dry milk in Tris-buffered saline (TBS) at room temperature for $1.5 \mathrm{~h}$, followed by overnight incubation of primary antibodies diluted in $5 \%$ BSA at $4^{\circ} \mathrm{C}$ on the shaker. Primary antibodies were rabbit anti-Kv1.3 (Thermo Fisher Scientific cat. no. PA5-77618, RRID:AB_2736055), rat anti-MBP (Abcam cat. no. ab7349, RRID:AB_305869), rabbit anti-phospho-AKT (p-AKT; Cell Signaling Technology cat. no. 4060, RRID:AB_2315049), rabbit anti-AKT (Cell Signaling Technology cat. no. 4691, RRID:AB_915783), rabbit anti-phospho-p38 mitogen-activated protein kinase (MAPK) (p-p38; Cell Signaling Technology cat. no. 4511, RRID:AB_2139682), rabbit anti-p38 MAPK (Cell Signaling Technology cat. no. 8690, RRID:AB_10999090), and mouse anti$\beta$-actin (Proteintech cat. no. 66009-1-lg, RRID:AB_2782959). Afterward, membranes were washed in TBS with $0.1 \%$ Tween20 (TBST) for $10 \mathrm{~min} \times$ three times and then incubated in diluted secondary antibody for $1 \mathrm{~h}$ at room temperature on a shaker. The secondary antibodies were horseradish peroxidase (HRP)-conjugated anti-rabbit (Jackson ImmunoResearch Labs cat. no. 111-035-003, RRID:AB_2313567), HRP-conjugated anti-rat (Jackson ImmunoResearch Labs cat. no. 112-035-003, RRID:AB_2338128) and HRP-conjugated anti-mouse secondary antibodies (Proteintech cat. no. SA00001-1, RRID:AB_2722565). After washing, membranes were finally incubated with ECL Western blot substrate (Solarbio) to detect HRP-conjugated secondary antibodies and imaged using the Image Lab system (Image Lab Software, RRID:SCR_014210). Band densities were measured by ImageJ software (ImageJ, RRID:SCR_003070).

\section{Statistical Analysis}

All data are expressed as mean \pm SE unless otherwise indicated. Statistical analyses were performed by one-way analysis of variance followed by a Fisher least-significant difference test for multiple comparisons. The difference between groups was considered significant at $P<0.05$.

\section{RESULTS}

\section{Alteration of Kv1.3 Expression in Ol Lineage Cell Development}

The expressions of MBP and Kv1.3 were first examined at different periods of development. Immunofluorescences showed the Kv1.3 protein decreased within the maturation. At the sixth day after cells were changed into OPCDM (DF 6d), the Kv1.3 immune density reduced approximately $30 \%$ of that in OPCs. At the eighth day in OPCDM (DF 8d), Kv1.3 immune density further decreased to $52.6 \% \pm 2.8 \%$ of OPCs (Figure 1A). Similar result was observed in Western blot, the Ol Kv1.3 expression decreased approximately 28 and 58\% when OPCs differentiated 6 and 8 days, respectively. In contrast to Kv1.3 expression, the $\mathrm{MBP}$ expression increased in the differentiating process. MBP started showing a significant increase after 6 days in OPCDM and further increased when the differentiating culture extended to 8 days (Figure 1B). MBP is a myelin structural protein that is widely used to illustrate the maturation and myelinating capability of Ols. Following the expression, we thought to test the function and activity of Kv1.3 in OPCs and Ols. Voltage clamp was performed to test the Kv1.3 currents in cultured cells. PAP $10 \mathrm{nM}\left(\mathrm{EC}_{50}=2 \mathrm{nM}\right.$; Schmitz et al., 2005) was perfused into the extracellular solution immediately after total currents were recorded to particularly suppress Kv1.3 currents, and then the Kv1.3-excluded currents were recorded at the same cell. The isolated Kv1.3 currents were achieved by subtraction of Kv1.3-excluded currents from total currents. Kv1.3 contributed a major component in the OPC outward $\mathrm{K}^{+}$currents as shown 
in the first line of Figure 1C, and the current density attenuated approximately 27.9, 47.7, and 65.1\% after 4 days (DF 4d), 6 days, and 8 days in OPCDM (Figure 1C). As the changes of Kv1.3 and MBP expressions both reached significant enhancement after 6 days in differentiating culture, the time point of day 6 was chosen for the following studies regarding mature Ols.

\section{Involvement of Kv1.3 in IL-17-Induced Inhibition of OPC Proliferation and Differentiation}

Cytokine IL-17 level is significantly elevated in MS patients CSF, and it is found to be associated with disease severity and progression in CNS demyelination model (Kostic et al., 2014). IL-17 is demonstrated to be enhancing oxidative stressinduced $\mathrm{Ol}$ apoptosis (Paintlia et al., 2011) and strongly inhibiting OPC differentiation (Kang et al., 2013). Here, we tested the consequence of IL-17 on OPCs and Ols. The dose of IL-17 was tittered by MTT assay performed in OPCs. IL-17 significantly decreased OPC viability to $83.40 \% \pm 4.5 \%$ at the concentration of $200 \mathrm{ng} / \mathrm{mL}$ and (Figure 2A, $P=0.0037$, IL-17 $200 \mathrm{ng} / \mathrm{mL}$ vs. control) further decreased to $75.5 \% \pm 5.9 \%$ at $400 \mathrm{ng} / \mathrm{mL}$ (Figure 2A, $P<0.0001$, IL-17 $400 \mathrm{ng} / \mathrm{mL}$ vs. control). Thus, the dose of $200 \mathrm{ng} / \mathrm{mL}$ was chosen for the following studies. Moreover, IL-17 up to $400 \mathrm{ng} / \mathrm{mL}$ did not affect the OPC apoptosis (data not shown) in our culture system. Previous studies reported that Kv1.3 on T lymphocytes and microglial cells play a key role in pathophysiological processes in inflammatory brains; we next explored whether OPC/Ol Kv1.3 was directly involved in inflammation-related myelin injury. Experiments were next performed to assess the expression of OPC Kv1.3 after exposure to IL-17. Exposure of IL-17 for $48 \mathrm{~h}$ enhanced the Kv1.3 protein expression in OPCs (Figure 2E, $P=0.0114$, IL-17 vs. control). PAP was preadded to culture medium $30 \mathrm{~min}$ prior to following addition of BrdU with or without IL-17 for $48 \mathrm{~h}$. PAP mitigated IL-17-induced OPC viability reduction (Figure 2B, $P=0.0007$, PAP + IL-17 vs. IL-17), and counteracted IL-17-caused decrease in $\mathrm{BrdU}^{+}$cells percentage (Figures 2C,D; $P<0.0001$, PAP + IL-17 vs. IL-17). These results together suggested IL-17 retarded the OPC proliferation at the concentration of $200 \mathrm{ng} / \mathrm{mL}$, and blockade of Kv1.3 prevented IL-17-caused loss of cell viability and inhibition of proliferation. Consistent results were found in differentiated Ols. MBP is an essential myelin structural protein, which is believed to indicate the $\mathrm{Ol}$ myelinating ability in vitro. MBP classically has three isoforms due to the different transcription start sites. The analysis described below was based on the protein bands at $18.5 \mathrm{kDa}$, which is considered as the predominant isoform essential for CNS myelin stability (Smith et al., 2012; Vassall et al., 2013). IL17 decreased the expression of MBP (Figures 2F,G; $P=0.0015$, control vs. IL-17) and increased the Ol Kv1.3 protein expression (Figures 2F,G; $P=0.0106$, control vs. IL-17). Pretreatment of PAP for $30 \mathrm{~min}$ counteracted MBP reduction (Figures 2F,G; $P=0.0013$, PAP + IL-17 vs. IL-17) and Kv1.3 enhancement (Figures 2F,G; $P=0.0082$, PAP + IL-17 vs. IL-17) caused by IL-17. These together suggested the involvement of Kv1.3 in IL17-induced inhibition of OPC proliferation and differentiation.

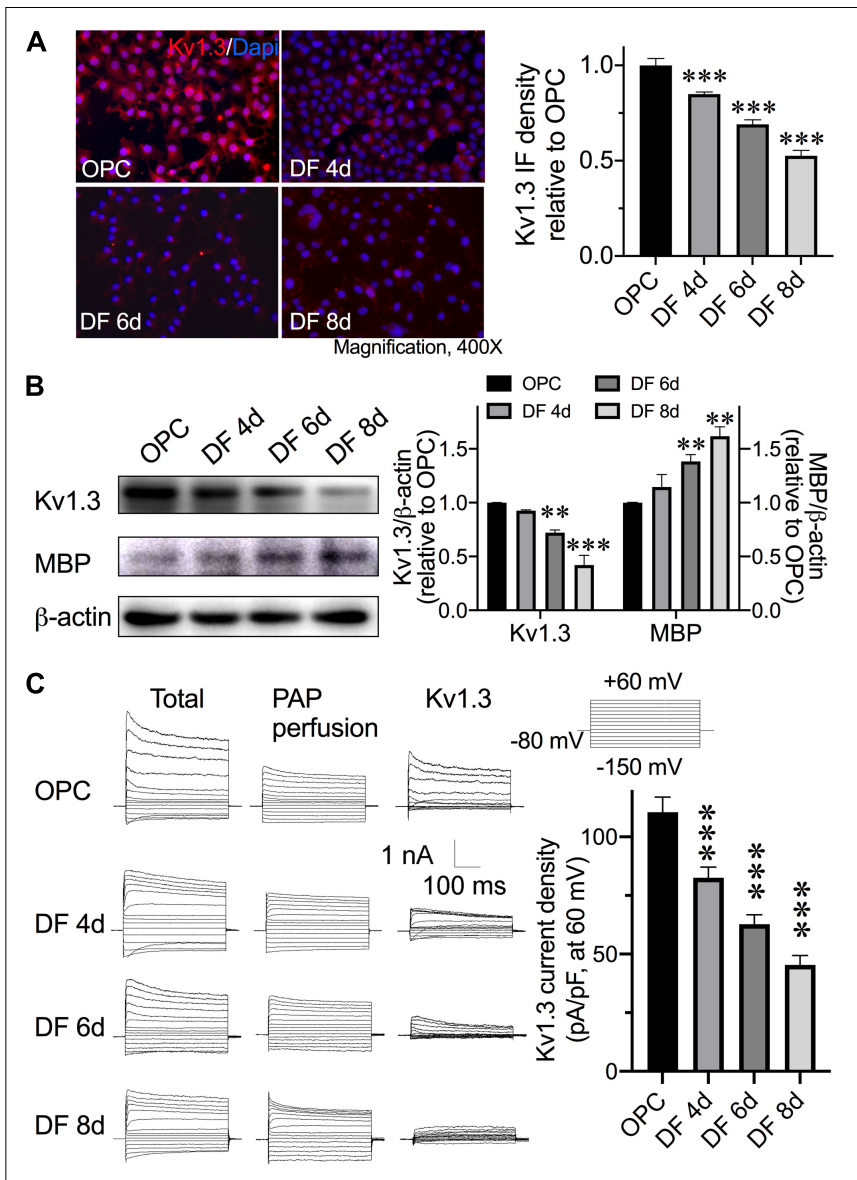

FIGURE 1 | Expression of Kv1.3 in OPCs/Ols during development. OPCs were cultured in OPCDM to differentiate into mature Ols. OPCs were transferred into OPCDM for 4 days (DF 4d), 6 days (DF 6d), and 8 days (DF 8d). (A) Representative images of Kv1.3 immunofluorescence staining (red) in cultures. Intact cell nuclei were visualized with DAPI (blue). The

immunofluorescence density of Kv1.3 was summarized in a bar graph at the right. With the maturity of OPCs, Kv1.3 decreased in a

development-dependent manner. (B) Western blot analysis of MBP expression in cells collected from different periods of OPCs/Ols. Band densitometry data are shown in the bar graph (right). Data are normalized to $\beta$-actin shown in each gel. In contrast to the Kv1.3 alterations, the expression of MBP increased with differentiation. (C) Representative current traces of outward $\mathrm{K}^{+}$currents recorded during depolarizing and hyperpolarizing pulses are shown in cells of OPCs, DF 4d, DF 6d, and DF 8d. The whole-cell outward $\mathrm{K}^{+}$currents recorded before (Total) and $15 \mathrm{~min}$ after superfusion of $10 \mathrm{nM}$ PAP (PAP perfusion) to the bath. The Kv1.3 currents were then isolated by subtraction of outward $\mathrm{K}^{+}$currents recorded in the presence of PAP from the total currents (Kv1.3). The summary bar graph illustrating average Kv1.3 current density measured at $+60 \mathrm{mV}(\mathrm{pA} / \mathrm{pF})$ obtained from OPCs and Ols ( $n$ =16). With the maturation of Ols, the Kv1.3-conducted potassium currents decreased. All data expressed were obtained from three independent experiments unless otherwise indicated. ${ }^{* *} P<0.01$ vs. control, ${ }^{* * *} P<0.001$ vs. control.

\section{Kv1.3 Involved in IL-17-Induced OPC Developmental Alterations by Diminishing AKT Signal}

Thus far, we have demonstrated IL-17 up-regulated Kv1.3 protein expression in OPCs and Ols, and blockade of Kv1.3 

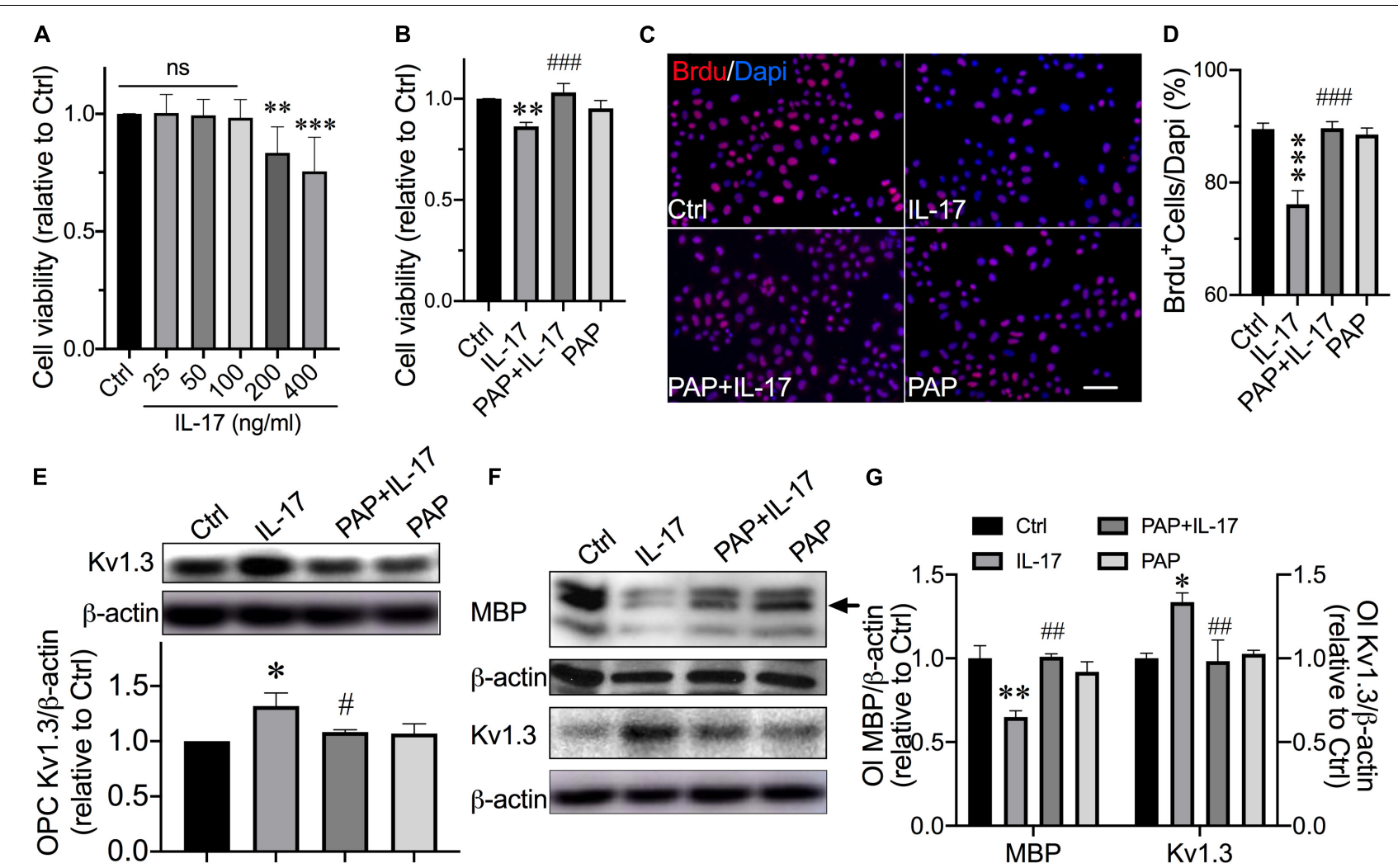

FIGURE 2 | Kv1.3 blockade prevented OPCs from IL-17-induced inhibition of proliferation and differentiation. OPCs were exposed to IL-17 (200 ng/mL) for $48 \mathrm{~h}$ with or without preaddition of PAP (10 nM) for $30 \mathrm{~min}$. (A) The dose of IL-17 was tittered by MTT assay performed in OPCs $(n=6)$. IL-17 significantly reduced cell viability at a concentration of $200 \mathrm{ng} / \mathrm{mL}$ and further reduced at the concentration of $400 \mathrm{ng} / \mathrm{mL}$. (B) MTT assay was performed to detect OPC viability $(n=7)$. PAP pretreatment counteracted the loss of cell viability induced by IL-17. (C) OPCs were treated with IL-17 with or without PAP in the presence of BrdU (10 $\mu \mathrm{M}$ ) for $48 \mathrm{~h}$ Representative images of merged BrdU immunofluorescence staining (red) and DAPI (blue) are shown. Scale bar $=20 \mu \mathrm{m}$. The average percentage of BrdU ${ }^{+}$cells from five independent experiments are summarized in (D) There were 10 randomly selected visual fields counted for each group from three independent experimental treatments. IL-17-induced reduction of BrdU+ cells was attenuated by PAP. (E) Western blot analysis of Kv1.3 expression in OPCs. Band densitometry data are shown in the bar graph (below) $(n=4)$. IL-17 treatment for $48 \mathrm{~h}$ elevated the Kv1.3 protein expression in OPCs, whereas the PAP attenuated this elevation. For experiments conducted with Ols in $(\mathbf{F}, \mathbf{G})$, Ols were exposed to IL-17 (200 ng/mL) with or without prior addition of PAP (10 nM) for $30 \mathrm{~min}$ during the differentiation culture in OPCDM for 6 days. (F,G) Representative images and statistical analyses of MBP and Kv1.3 expression in mature Ols of Western blot $(n=3)$. The band marked by the arrow is a protein band with a size of $18.5 \mathrm{kDa}$. Pretreatment with PAP for $30 \mathrm{~min}$ counteracted the decrease in MBP and increase in Kv1.3 protein expression in Ols induced by IL-17. ${ }^{*} P<0.05$ vs. control, ${ }^{* *} P<0.01$ vs. control, ${ }^{* * *} P<0.001$ vs. control. $\# P<0.05$ vs. IL-17, \#\# $P<0.01$ vs. IL-17, $\# \# P<0.001$ vs. IL-17.

mitigated IL-17-induced OPC proliferation and differentiation retardation. The mechanisms of Kv1.3 involvement in IL-17caused alterations of OPC proliferation and differentiation attracted our attention. It has been shown that OPC differentiation is controlled by a number of factors, many of which act to be inhibitory including leucine-rich repeat and immunoglobulin domain-containing 1 (Mi et al., 2004, 2005), Notch-1 (Bongarzone et al., 2000; Kim et al., 2008), Wnt (Shimizu et al., 2005) etc., whereas p38 MAPK (Bhat et al., 2007; Chew et al., 2010) and AKT (Flores et al., 2008) have been shown to be required for OPC differentiation and myelination. Previous studies reported that IL-17 increased total p38 levels and restricted neural stem cell differentiating to neurons, astrocytes, and OPCs (Li et al., 2013). AKT signal is reported to be protective for myelination under IL-17-enriched conditions (Tsiperson et al., 2013; Liu et al., 2017b). We next sought to clarify whether Kv1.3 regulated OPC proliferation and differentiation through AKT and/or p38 pathways. The phosphorylation forms of p38 (p-p38) and AKT (p-AKT) proteins were examined to illustrate the activation level of these signals. IL-17 decreased the expression of $\mathrm{p}$-AKT in OPCs (Figure 3A; $P=0.0074$, IL-17 vs. control) and differentiated Ols (Figure 3B; $P=0.0058$, IL-17 vs. control) but did not alter the activation of p-p38 in OPCs (Figure 3A; $P=0.94$ ) and Ols (Figure 3B; $P=0.54$ ). In both OPCs and mature Ols, PAP prevented p-AKT from IL-17-caused reduction (PAP + IL-17 vs. IL-17, $P=0.0297$ in Figure 3A and $P=0.0449$ in Figure 3B). These results suggested Kv1.3 may be involved in IL-17-induced inhibition of OPC proliferation and differentiation by diminishing AKT signal pathways but not p38.

By knowing the contribution of AKT inactivation in Kv1.3 involvement of IL-17-induced OPC cell cycle alterations, a highly selective, efficient, and cell-permeable AKT activator SC79 (Jo et al., 2012) was employed to convince the contribution of AKT. We first confirmed the efficiency of SC79 on AKT activation 

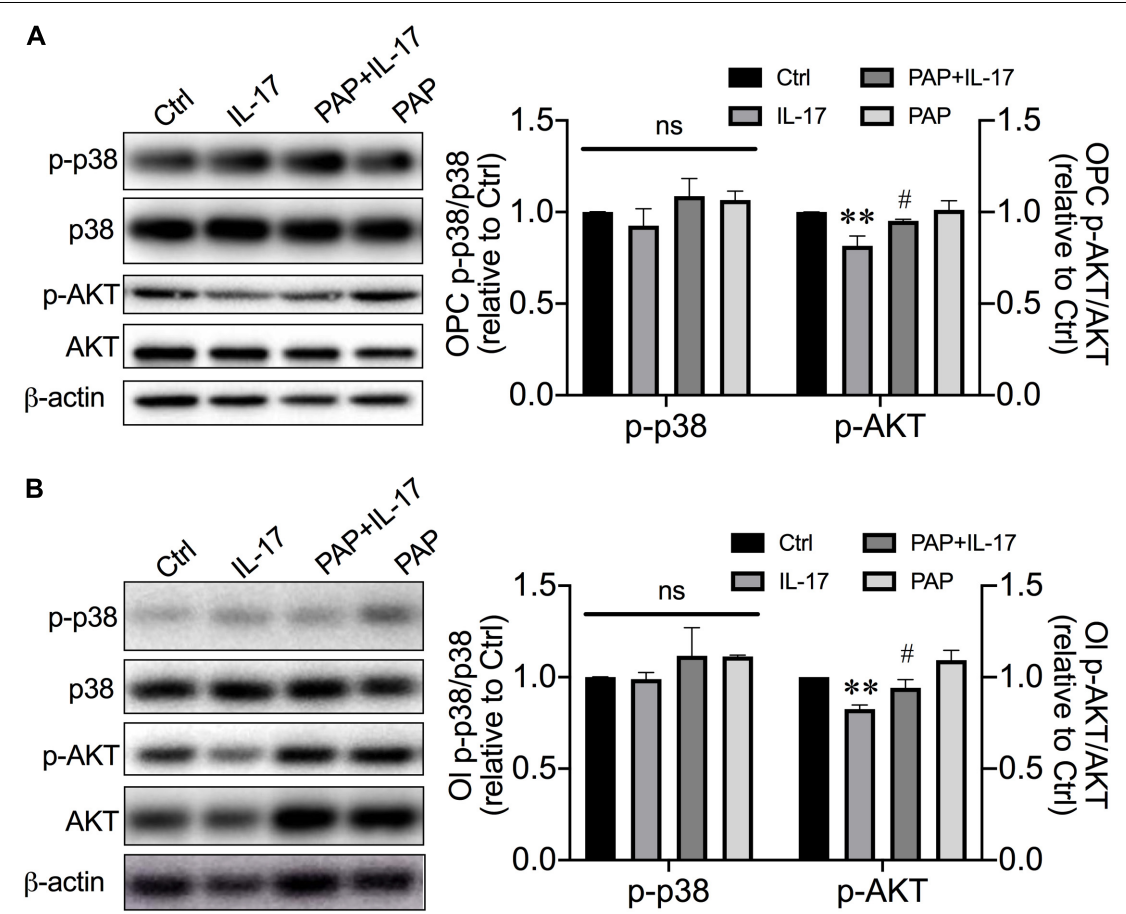

FIGURE 3 | Kv1.3 involved in IL-17-induced developmental alterations by diminishing AKT signal but not p38 MAPK. OPCs were treated as described in Figure 2. (A) Representative images and statistical analyses of p-p38 and p-AKT expression in OPCs of Western blot $(n=3)$. IL-17 suppressed the p-AKT expression, which represented the activation level of AKT pathway in OPCs, but not p-38, whereas PAP relieved this suppression. (B) For experiments conducted with Ols, OPCs were transferred into OPCDM and treated the same as described in Figure 2. Representative images and band densitometry data (right) of p-p38 and p-AKT expression in Ols $(n=4)$. The attenuation of p-AKT induced by IL-17 was mitigated by PAP, indicating the integral role of Kv1.3 in IL-17-caused decline of AKT activation. ${ }^{* *} P<0.01$ vs. control, ${ }^{\#} P<0.05$ vs. IL-17.

in our culture system after a 6-day application (Figure 4E; $P=0.018$, SC79 vs. control). For cotreatment, SC79 was applied to culture medium at the same time with addition of IL-17. With the presence of IL-17, SC79 also performed effectively on AKT activation (Figure 4E; $P=0.0094$, IL17 vs. SC79 + IL-17). SC79 showed the protective effects on OPC proliferation (Figures 4A-C) and Ol MBP expression (Figure 4D). Application of SC79 protected OPCs from IL17 -induced loss of viability (Figure 4A; $P=0.0093$, IL-17 vs. SC79 + IL-17) and mitigated IL-17-caused BrdU ${ }^{+}$cell number decrease (Figures 4B,C; $P=0.0057$, IL-17 vs. SC79 + IL-17). In differentiating culture, SC79 protected Ols from IL-17-induced attenuation of MBP expression (Figure 4D; $P=0.0014$, IL-17 vs. SC79 + IL-17) and mitigated IL-17-caused Kv1.3 elevation (Figure 4F; $P<0.0001$, IL-17 vs. SC79 + IL-17). These results suggested that AKT activation promotes the OPC proliferation and differentiation in exposure of IL-17, and decreased AKT activation participated in Kv1.3 involvement of IL-17-induced inhibition of OPC proliferation and differentiation.

\section{Kv1.3 Blockade Prevented Ols From LPC-Induced Myelin Loss in vivo}

Having observed the MBP expression altered in cell culture, the LPC-induced demyelination mice model was utilized to interrogate myelin repair in conditions more comparable with human disease to directly disclose the effects of Kv1.3 and its blockade on myelin sheath. The LPC-induced experimental demyelination is advantageous for characterizing the focal myelin morphology because the location of the damage is known. Because corpus callosum is a sensitive marker for damage of the cerebral white matter demyelination, the areas of corpus callosum were chosen to inject LPC and observe myelination. According to the previous publication, PAP distributes in parallel in plasma and brain following enteral administration and showed even higher concentration in most tested brain regions than in plasma (Maezawa et al., 2018). The plasma concentrations following $6 \mathrm{mg} / \mathrm{kg}$ i.p. injection achieved $300 \mathrm{nM}$ after $8 \mathrm{~h}$ and still above $10 \mathrm{nM}$ after $24 \mathrm{~h}$ (Azam et al., 2007). Considering the PAP $\mathrm{EC}_{50}=2 \mathrm{nM}$ (Schmitz et al., 2005), a predicable concentration of greater than $10 \mathrm{nM}$ is sufficient to achieve pharmacological Kv1.3 blockade in mice brains. We decided to administer PAP by i.p. injection at a dose of $6 \mathrm{mg} / \mathrm{kg}$ every $24 \mathrm{~h}$. Demyelination lesions in the corpus callosum were revealed by the LFB method, which stains myelin in blue. Myelin showed intact appearance in the injection point of sham animals, while it appeared discontinuous and reduced significantly in overall density of staining in LPC-injected sites. PAP significantly improved the myelin integrity compared with that in LPCinjected corpus callosum (Figure 5A). Myelination was also evaluated by blotting reactivity of MBP. The tissue of injection areas was taken to examine $\mathrm{MBP}$ expression. In agreement 
A

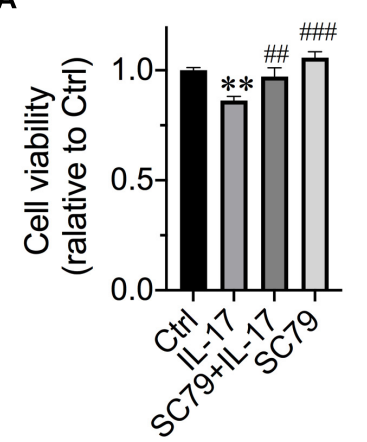

B

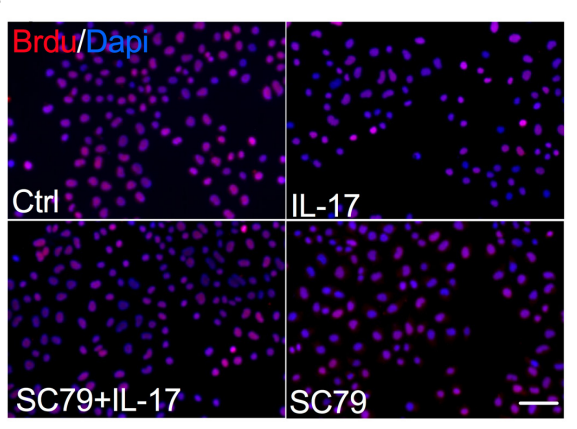

C

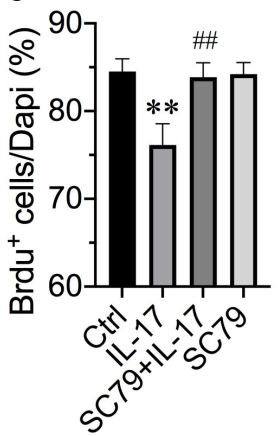

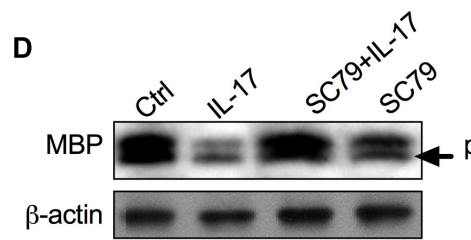

E
p-AKT
AKT
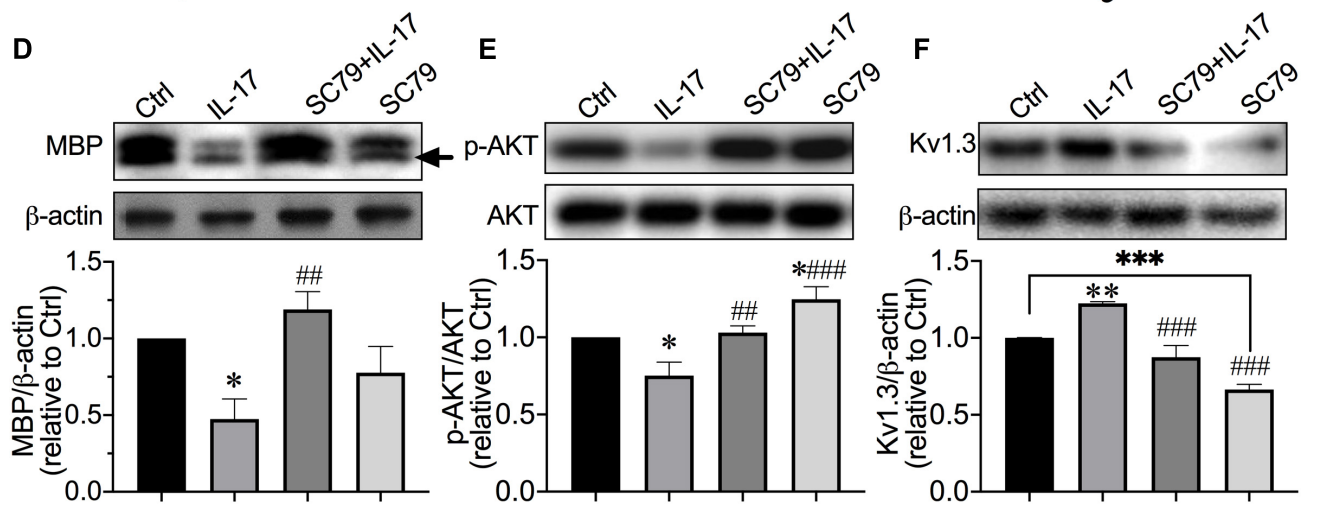

FIGURE 4 | Protection of AKT activator on IL-17-induced inhibition of OPC proliferation and differentiation. OPCs were exposed to IL-17 (200 ng/mL) for $48 \mathrm{~h}$ with or without AKT activator SC79 (10 $\mu \mathrm{M})$. (A) MTT assay was performed to detect OPC viability $(n=7)$. SC79 counteracted the decrease in cell viability induced by IL-17. (B) OPCs were treated as described before in the presence of $\mathrm{BrdU}(10 \mu \mathrm{M})$ for $48 \mathrm{~h}$. Representative images of merged BrdU immunofluorescence staining (red) and DAPI (blue) are shown. Scale bar $=20 \mu \mathrm{m}$. The average percentage of $\mathrm{BrdU}^{+}$cells from five independent experiments are summarized in (C) There were 10 randomly selected visual fields counted for each experimental group from three independent treatments. IL-17-induced reduction of BrdU ${ }^{+}$cell percentage was attenuated by SC79. For experiments conducted with Ols in (D-F), OPCs were exposed to IL-17 (200 ng/mL) with or without SC79 (10 $\mu$ M) in OPCDM for 6 days. (D-F) Representative images and statistical analyses of MBP $(n=4)$, p-AKT $(n=4)$, and Kv1.3 $(n=5)$ expressions in Ols of Western blot. Band densitometry data are shown in the bar graph (below). SC79 effectively activated AKT signal in our culture system. Similar to the PAP, SC79 counteracted the IL-17-induced decrease in MBP and increase in Kv1.3 expression and mitigated the decrease in $\mathrm{p}-\mathrm{AKT}$ induced by IL-17. ${ }^{*} P<0.05$ vs. control, ${ }^{* *} P<0.01$ vs. control, ${ }^{* * *} P<0.001$ vs. control. \#\#P<0.01 vs. IL-17, \#\#\# $<0.001$ vs. IL-17.

with the morphological observation, LPC decreased the MBP expression to approximately $36 \%$ of sham (Figure 5B; $P<0.0001$, LPC vs. sham), and this reduction was not observed in Kv1.3 inhibitor/LPC-cotreated mice (Figure 5B; $P<0.0001$, LPC vs. PAP + LPC). By exploring the alterations of myelination, the OPC proliferation was next examined in mice. In LPCtreated mice, the number of $\mathrm{BrdU}^{+} \mathrm{NG}^{+}$cells were significantly lessened compared with sham mice (Figure 5C; $P=0.0147$, LPC vs. sham), and administration of PAP prevented the decrease in number of $\mathrm{BrdU}^{+} \mathrm{NG}^{+}$cells (Figure 5C; $P=0.0147$, LPC vs. PAP + LPC). These results together suggested that Kv1.3 blockade is protective for OPC proliferation and myelination in vivo.

\section{DISCUSSION}

In the present study, we demonstrated that Kv1.3 blockade effectively promoted OPC proliferation and differentiation through activation of AKT signaling, leading to protection of myelin from IL-17- and LPC- induced myelin damage in vitro and in vivo, which is beneficial to remyelination in neurological disorders with demyelination.

IL-17-induced $\mathrm{Ol}$ injury is closely relative to MS pathogenesis. MS is the most common cause of non-traumatic disability in young people, affecting approximately 2.5 million people worldwide (Pugliatti et al., 2002; Compston and Coles, 2008). Axonal remyelination has been a challenge in MS therapies. To date, there is no proven way to repair demyelinated lesions. Disease progression usually has two phases: relapsing-remitting phase and progressive phase. The different disease phases reflect different underlying neuropathology, with inflammation and demyelination in the relapsing-remitting phase and neurodegeneration in the progressive phase (Jolanda Munzel and Williams, 2013). Previous studies of Kv1.3 in MS and other neuroinflammatory diseases were mainly focused on the ionic immune regulating role of Kv1.3 in T cells (Rus et al., 2005; Schmitz et al., 2005; Eil et al., 2016; Wang et al., 2019) and microglia (Fordyce et al., 2005; Di Lucente et al., 2018), which were relative to the pathogenesis in relapsingremitting phase. Here we demonstrated a direct toxic effect of IL-17-induced Kv1.3 overexpression on myelin-producing 


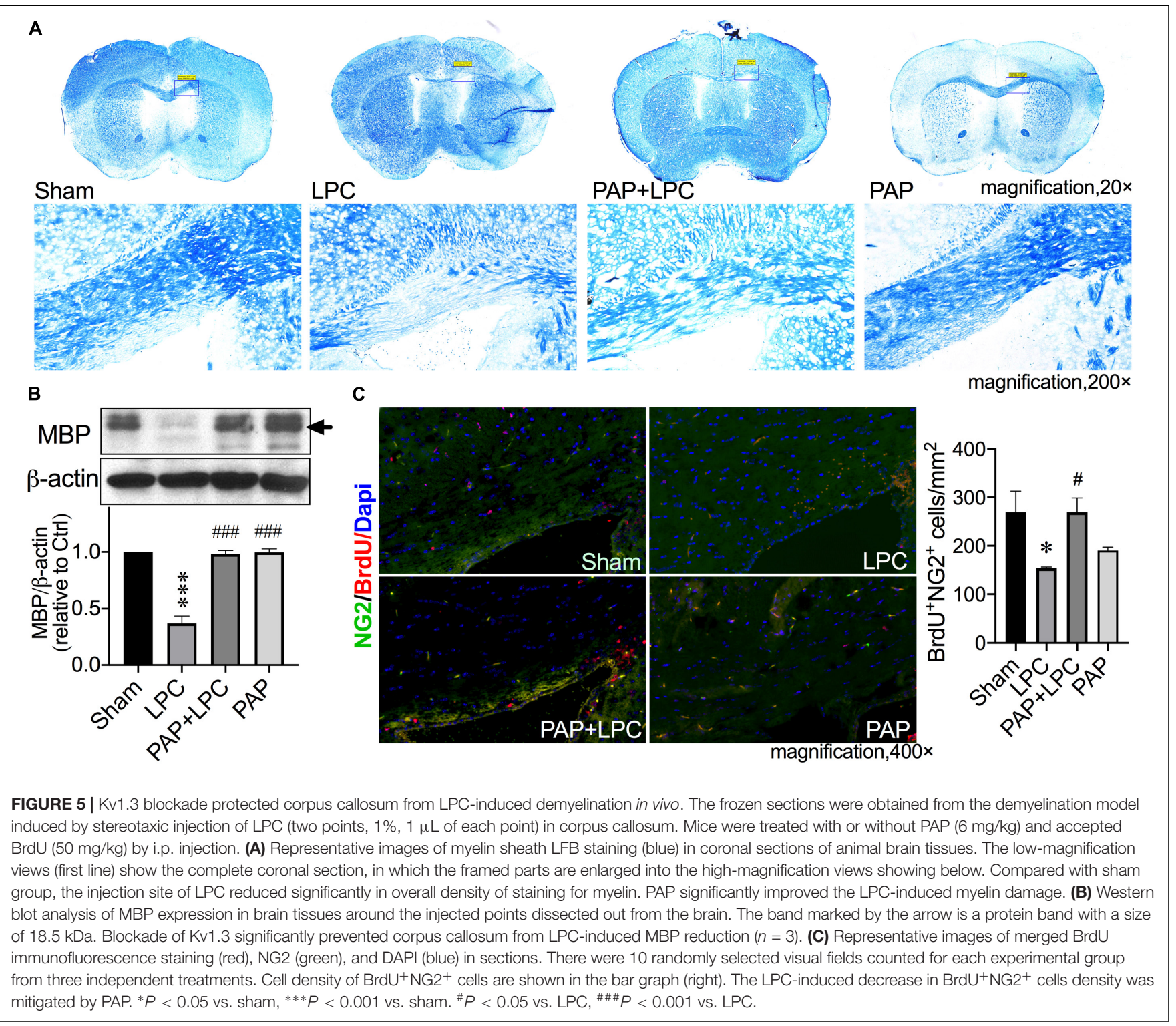

cells OPCs and Ols. Such a direct toxicity was validated by using a relatively immune-independent mouse model that maximally eliminates the probable involvement of T-cell Kv1.3 in observed results. Myelin damage causes further axonal injury related to neurodegeneration in the progressive phase, because myelinated axons appear myelin dependency during development (Alizadeh et al., 2015). Additionally, an increased number of IL-17-expressing cells were observed in ischemic brain tissues of both human and rodent ( $\mathrm{Li}$ et al., 2005; Gelderblom et al., 2012), suggesting that IL-17 participates in the CNS pathophysiology of secondary inflammation after ischemic stroke. The MS appears to be the disease mostly associated with IL-17, although IL-17 is relatively associated with different inflammatory conditions in the CNS.

IL-17-producing subset of $\mathrm{CD}^{+}{ }^{+} \mathrm{T}$ cells are identified as Th17 cells. The role of Th17 cells in the pathogenesis of relapsing-remitting MS has been demonstrated in several studies, showing the identification of Th17 cells in the MS lesions but not in normal-appearing white matter tissues or control brain specimens (Tzartos et al., 2008) and increased levels of Th17 cell gene expression as well as IL-17 protein in MS brain lesions (Lock et al., 2002; Komiyama et al., 2006; Tao et al., 2014). Although at certain conditions IL-17 may boost the differentiation of OPCs (Rodgers et al., 2015), most publications reported that IL-17 was harmful for OPC and Ol survival and function (Paintlia et al., 2011; Li et al., 2013; Waisman et al., 2015; Wang et al., 2017), thus promoting inflammatory injuries. Our results disclosed that IL-17 caused OPC loss of cell viability at the concentrations of $200 \mathrm{ng} / \mathrm{mL}$ or greater. Together with the results from BrdU experiments, we demonstrated that IL17 inhibited OPC proliferation (Figure 2) without alteration of cell apoptosis (data not shown), which is consistent with previous results obtained with primary OPC culture (Rodgers et al., 2015). Similar results were also observed in neural stem cells that IL-17 
stimulated neural stem cell way out of cell cycle despite no change of apoptosis (Li et al., 2013). Controversial results were reported in primary OPCs cultured with IL-17 for 4 days at the concentrations of 50 and $200 \mathrm{ng} / \mathrm{mL}$ (Kang et al., 2013). We next examined the effect of IL-17 on OPC differentiation. Application of IL-17 into OPCDM for 6 days at a concentration of $200 \mathrm{ng} / \mathrm{mL}$ inhibited the OPC differentiation (Figure 2). Kang et al. claimed an inhibitory effect on OPC maturation of $25 \mathrm{ng} / \mathrm{mL}$ IL-17 addition to culture medium with absence of proliferative signal platelet-derived growth factor (PDGF) (Kang et al., 2013). Rodgers et al. observed an enhancement of OPC differentiation in both absence and presence of PDGF, while culture treated with IL-17 at concentrations of 25 and $50 \mathrm{ng} / \mathrm{mL}$ within the first $2 \mathrm{~h}$ of plating (Rodgers et al., 2015). These contrast findings suggest the role of IL-17 on OPC differentiation is highly time- and dose-dependent. The discrepancies may also attribute to the culture system and stage of cells within the Ol lineage.

Kv1.3 is highly expressed in postmortem MS brain plaques, localized on inflammatory infiltrates (Rus et al., 2005) and OPCs (Tegla et al., 2011). These findings indicate the involvement of Kv1.3 in MS pathogenesis. Indeed, several groups reported the contribution of Kv1.3 in MS-related pathogenesis, and most data were from inflammatory cells in experimental autoimmune encephalomyelitis (EAE) model. Blockade of Kv1.3 revealed beneficial effects on alleviation of EAE (Huang et al., 2017; Fan et al., 2018; Yuan et al., 2018; Zhao et al., 2020). Preclinical trials targeting at Kv1.3 to treat autoimmune disease, including neuroinflammatory MS, were conducted and showed positive results (Perez-Verdaguer et al., 2016; Prentis et al., 2018). Although it is known that Kv1.3 is expressed on OPCs in MS lesion, the role of OPC/Ol Kv1.3 in MS pathogenesis is less appreciated. We showed that Kv1.3 currents and channel expression decreased during the OPC development in the cell culture (Figure 1), which is in agreement with previous observation on primary cultures (Chittajallu et al., 2002). IL17-induced reduction of OPC proliferation and MBP expression in cultures were prevented by Kv1.3 antagonist, but antagonist alone did not alter proliferation and MBP expression (Figure 2), suggesting that Kv1.3 is involved in MS-related myelin damage, and Kv1.3 blockade affects OPC cell cycle particularly under pathological conditions. This may explain the controversial results from Vautier et al. (2004) that overexpression of Kv1.3 enhanced OPC proliferation but did not interfere differentiation when conducted under relatively physical conditions. Although we demonstrated an enhancement of Kv1.3 expression after IL-17 treatment, the molecular mechanisms remain obscure. In microglial cells, Kv1.3 expression is elevated by ERK1/2 activation (Liu et al., 2013), which is downstream molecular of IL-17. IL-17 may increase Kv1.3 expression potentially by activating ERK1/2 signal. AKT (Flores et al., 2008) and p38 MAPK (Bhat et al., 2007; Chew et al., 2010) are the main positive signals for OPC development. It is reported that AKT phosphorylation is dependent on Kv1.3 activation in Ol lineage cells and situated downstream of Kv1.3 (Tegla et al., 2011), and coexpression of PKB/AKT with Kv1.3 in oocytes enhanced the Kv1.3 channel abundance, suggesting AKT up-regulates
Kv1.3 expression (Warsi et al., 2015). In both natural killer cells and T cells, Kv1.3 seems to activate AKT/mTOR signal cascade (Eil et al., 2016; Geng et al., 2020). Together, these findings suggest that Kv1.3 and AKT act synergistically. We examined the involvement of p38 MAPK and AKT in Kv1.3mediated reduction of OPC proliferation and differentiation. The activation of AKT but not p38 MAPK was inhibited by IL-17-induced Kv1.3 enhancement, which was counteracted by AKT activator SC79 (Figures 3, 4). This is a novel pattern different from previously demonstrated synergism of Kv1.3 and AKT. It is worth pointing out that downstream molecules of IL-17, including but not limited to ERK (Amatya et al., 2017; Xie et al., 2020; Allan et al., 2021), JNK (Amatya et al., 2017; Milovanovic et al., 2020), and nuclear factor $\kappa \mathrm{B}$ (Jiang et al., 2017; Wu et al., 2018), may also contribute to the observed alterations of OPC cell cycle caused by IL-17, which were not examined in the present study. In addition, studying the role of the aforementioned negative signals in OPC development may be needed in the future, which will help us better understand the regulatory complex.

EAE is the most utilized animal model to explore MSrelated pathogenesis and therapeutic strategy. The current immunosuppressive therapies are mainly based on the research done with EAE. Indeed, EAE is very heterogeneous regarding induction methods, clinical and pathological features, and amenability to treatments. The idea of EAE is based on the hypothesis that MS is mediated by autoreactive T-cell infiltration into CNS. But this hypothesis is challenged by studies that demonstrate occurrence of $\mathrm{Ol}$ death as the very early and perhaps the initial event in the pathology of the plaque, even before development of inflammation (Barnett and Prineas, 2004; Trapp, 2004). This brings EAE limitations when applied to human disease (Sriram and Steiner, 2005; Gold et al., 2006), particularly for remyelination. However, we focused on the OPC/Ol Kv1.3 function in inflammatory brains; LPC-induced demyelination was chosen to minimize the role of infiltrated T-cell Kv1.3 in the present study. Each model has its advantages and limitations. Other elements cannot be fully eliminated in LPC model, for example, the resident microglia around lesion site (Chen et al., 2020), which also expresses Kv1.3. Global administration of PAP may have the potential to inhibit Kv1.5 activity. However, the selectivity of PAP for Kv1.3 is approximately 23 -fold higher than Kv1.5 (Schmitz et al., 2005; Vicente et al., 2006), making the possibility of Kv1.5 activation ignorable. In addition, systemic application of PAP to animals may also target on neuronal and microglial Kv1.3 channels; such a potential might be minimal as only small amounts of Kv1.3 are expressed on neurons in some brain regions.

In summary, our current data showed that IL-17 induced inhibition of OPC proliferation and differentiation through Kv1.3 channel, resulting in retardation in axonal remyelination and consequent brain white matter damage. We, for the first time, demonstrated that the integral role of Kv1.3 in IL-17 caused ATK signal decline, resulting in inhibitory effects of OPC proliferation and differentiation. Collectively, these findings serve to define the pathogenesis of CNS inflammation-induced myelin damage and support a potential therapeutic strategy of using 
Kv1.3 blocker to treat MS and white matter damage in other neurological disorders.

\section{DATA AVAILABILITY STATEMENT}

The raw data supporting the conclusions of this article will be made available by the authors, without undue reservation.

\section{ETHICS STATEMENT}

The animal study was reviewed and approved by the Life Science Ethics Review Committee of Zhengzhou University.

\section{AUTHOR CONTRIBUTIONS}

HL conceived and designed the project and carried out experiments together with XY. YW and YY participated in collating the experimental date and analysis. HL, XY, JY, and RZ

\section{REFERENCES}

Alizadeh, A., Dyck, S. M., and Karimi-Abdolrezaee, S. (2015). Myelin damage and repair in pathologic CNS: challenges and prospects. Front. Mol. Neurosci. 8:35. doi: 10.3389/fnmol.2015.00035

Allan, K. C., Hu, L. R., Scavuzzo, M. A., Morton, A. R., Gevorgyan, A. S., Cohn, E. F., et al. (2021). Non-canonical targets of HIF1a impair oligodendrocyte progenitor cell function. Cell Stem Cell 28:e211.

Amatya, N., Garg, A. V., and Gaffen, S. L. (2017). IL-17 Signaling: the Yin and the Yang. Trends Immunol. 38, 310-322. doi: 10.1016/j.it.2017. 01.006

Azam, P., Sankaranarayanan, A., Homerick, D., Griffey, S., and Wulff, H. (2007). Targeting effector memory T cells with the small molecule Kv1.3 blocker PAP1 suppresses allergic contact dermatitis. J. Invest. Dermatol. 127, 1419-1429. doi: $10.1038 /$ sj.jid.5700717

Barnett, M. H., and Prineas, J. W. (2004). Relapsing and remitting multiple sclerosis: pathology of the newly forming lesion. Ann. Neurol. 55, 458-468. doi: 10.1002/ana.20016

Bhat, N. R., Zhang, P., and Mohanty, S. B. (2007). p38 MAP kinase regulation of oligodendrocyte differentiation with CREB as a potential target. Neurochem. Res. 32, 293-302. doi: 10.1007/s11064-006-9274-9

Bongarzone, E. R., Byravan, S., Givogri, M. I., Schonmann, V., and Campagnoni, A. T. (2000). Platelet-derived growth factor and basic fibroblast growth factor regulate cell proliferation and the expression of notch-1 receptor in a new oligodendrocyte cell line. J. Neurosci. Res. 62, 319-328. doi: 10.1002/10974547(20001101)62:3<319::aid-jnr1>3.0.co;2-g

Boulanger, J. J., and Messier, C. (2014). From precursors to myelinating oligodendrocytes: contribution of intrinsic and extrinsic factors to white matter plasticity in the adult brain. Neuroscience 269, 343-366. doi: 10.1016/j. neuroscience.2014.03.063

Boyd, A., Zhang, H., and Williams, A. (2013). Insufficient OPC migration into demyelinated lesions is a cause of poor remyelination in MS and mouse models. Acta Neuropathol. 125, 841-859. doi: 10.1007/s00401-013$1112-y$

Chang, A., Tourtellotte, W. W., Rudick, R., and Trapp, B. D. (2002). Premyelinating oligodendrocytes in chronic lesions of multiple sclerosis. N. Engl. J. Med. 346, 165-173. doi: 10.1056/nejmoa010994

Chen, M., Yang, L. L., Hu, Z. W., Qin, C., Zhou, L. Q., Duan, Y. L., et al. (2020). Deficiency of microglial Hvl channel is associated with activation of autophagic pathway and ROS production in LPC-induced demyelination mouse model. J. Neuroinflamm. 17:333. edited the manuscript. HX and YX contributed to the revision of the manuscript and gave pertinent opinions. All authors contributed to the article and approved the submitted and final published version.

\section{FUNDING}

This work was supported by the Chinese National Natural Science Foundation grant 81701247 awarded to HL. Furthermore, this study was also funded by the National Natural Science Foundation of China (Grant U190420029, 91849115 and 81530037 to YX) and the National Key R\&D Program of China (Grant 2017YFA0105003 to YX).

\section{ACKNOWLEDGMENTS}

We would like to express our heartfelt thanks to Yuzhen Liu of the First Affiliated Hospital of Xinxiang Medical University for her kindly help in the experiments.

Chew, L. J., Coley, W., Cheng, Y., and Gallo, V. (2010). Mechanisms of regulation of oligodendrocyte development by p38 mitogen-activated protein kinase. J. Neurosci. 30, 11011-11027. doi: 10.1523/jneurosci.2546-10.2010

Chittajallu, R., Chen, Y., Wang, H., Yuan, X., Ghiani, C. A., Heckman, T., et al. (2002). Regulation of Kv1 subunit expression in oligodendrocyte progenitor cells and their role in G1/S phase progression of the cell cycle. Proc. Natl. Acad. Sci. US A. 99, 2350-2355. doi: 10.1073/pnas.042698399

Compston, A., and Coles, A. (2008). Multiple sclerosis. Lancet 372, 1502-1517.

Dai, X., Qu, P., and Dreyfus, C. F. (2001). Neuronal signals regulate neurotrophin expression in oligodendrocytes of the basal forebrain. Glia 34, 234-239. doi: 10.1002 /glia.1057

Di Lucente, J., Nguyen, H. M., Wulff, H., Jin, L. W., and Maezawa, I. (2018). The voltage-gated potassium channel Kv1.3 is required for microglial proinflammatory activation in vivo. Glia 66, 1881-1895. doi: 10.1002/glia.23457

Eil, R., Vodnala, S. K., Clever, D., Klebanoff, C. A., Sukumar, M., Pan, J. H., et al. (2016). Ionic immune suppression within the tumour microenvironment limits T cell effector function. Nature 537, 539-543. doi: 10.1038/nature19364

Fan, C., Long, R., You, Y., Wang, J., Yang, X., Huang, S., et al. (2018). A novel PADRE-Kv1.3 vaccine effectively induces therapeutic antibodies and ameliorates experimental autoimmune encephalomyelitis in rats. Clin. Immunol. 193, 98-109. doi: 10.1016/j.clim.2018.02.012

Flores, A. I., Narayanan, S. P., Morse, E. N., Shick, H. E., Yin, X., Kidd, G., et al. (2008). Constitutively active Akt induces enhanced myelination in the CNS. J. Neurosci. 28, 7174-7183. doi: 10.1523/jneurosci.0150-08.2008

Fordyce, C. B., Jagasia, R., Zhu, X., and Schlichter, L. C. (2005). Microglia Kv1.3 channels contribute to their ability to kill neurons. J. Neurosci. 25, 7139-7149. doi: 10.1523/jneurosci.1251-05.2005

Gelderblom, M., Weymar, A., Bernreuther, C., Velden, J., Arunachalam, P., Steinbach, K., et al. (2012). Neutralization of the IL-17 axis diminishes neutrophil invasion and protects from ischemic stroke. Blood 120, 3793-3802. doi: 10.1182/blood-2012-02-412726

Geng, J., Wang, Y., Zhang, L., Wang, R., Li, C., Sheng, W., et al. (2020). The cajanine derivative LJ101019C regulates the proliferation and enhances the activity of NK cells via Kv1.3 channel-driven activation of the AKT/mTOR pathway. Phytomedicine 66:153113. doi: 10.1016/j.phymed.2019.153113

Gocke, A. R., Lebson, L. A., Grishkan, I. V., Hu, L., Nguyen, H. M., Whartenby, K. A., et al. (2012). Kv1.3 deletion biases T cells toward an immunoregulatory phenotype and renders mice resistant to autoimmune encephalomyelitis. J. Immunol. 188, 5877-5886. doi: 10.4049/jimmunol.1103095

Gold, R., Linington, C., and Lassmann, H. (2006). Understanding pathogenesis and therapy of multiple sclerosis via animal models: 70 years of merits and 
culprits in experimental autoimmune encephalomyelitis research. Brain 129, 1953-1971. doi: 10.1093/brain/awl075

Grishkan, I. V., Tosi, D. M., Bowman, M. D., Harary, M., Calabresi, P. A., and Gocke, A. R. (2015). Antigenic stimulation of Kv1.3-Deficient th cells gives rise to a population of Foxp3-Independent $\mathrm{T}$ cells with suppressive properties. J. Immunol. 195, 1399-1407. doi: 10.4049/jimmunol.1403024

Hauser, S. L., Chan, J. R., and Oksenberg, J. R. (2013). Multiple sclerosis: prospects and promise. Ann. Neurol. 74, 317-327. doi: 10.1002/ana.24009

Huang, J., Han, S., Sun, Q., Zhao, Y., Liu, J., Yuan, X., et al. (2017). Kv1.3 channel blocker (ImKTx88) maintains blood-brain barrier in experimental autoimmune encephalomyelitis. Cell Biosci. 7:31.

Jiang, M., Wu, Y. L., Li, X., Zhang, Y., Xia, K. L., Cui, B. W., et al. (2017). Oligomeric proanthocyanidin derived from grape seeds inhibited NF-kappaB signaling in activated HSC: involvement of JNK/ERK MAPK and PI3K/Akt pathways. Biomed. Pharmacother. 93, 674-680. doi: 10.1016/j.biopha.2017.06.105

Jo, H., Mondal, S., Tan, D., Nagata, E., Takizawa, S., Sharma, A. K., et al. (2012). Small molecule-induced cytosolic activation of protein kinase Akt rescues ischemia-elicited neuronal death. Proc. Natl. Acad. Sci. U S A. 109, 1058110586. doi: 10.1073/pnas. 1202810109

Jolanda Munzel, E., and Williams, A. (2013). Promoting remyelination in multiple sclerosis-recent advances. Drugs 73, 2017-2029. doi: 10.1007/s40265-0130146-8

Kang, Z., Wang, C., Zepp, J., Wu, L., Sun, K., Zhao, J., et al. (2013). Act1 mediates IL-17-induced EAE pathogenesis selectively in NG2+ glial cells. Nat. Neurosci. 16, 1401-1408. doi: 10.1038/nn.3505

Kim, H., Shin, J., Kim, S., Poling, J., Park, H. C., and Appel, B. (2008). Notchregulated oligodendrocyte specification from radial glia in the spinal cord of zebrafish embryos. Dev. Dyn. 237, 2081-2089. doi: 10.1002/dvdy.21620

Koch Hansen, L., Sevelsted-Moller, L., Rabjerg, M., Larsen, D., Hansen, T. P., Klinge, L., et al. (2014). Expression of T-cell KV1.3 potassium channel correlates with pro-inflammatory cytokines and disease activity in ulcerative colitis. J. Crohns Colitis 8, 1378-1391. doi: 10.1016/j.crohns.2014.04.003

Kolbinger, F., Huppertz, C., Mir, A., and Padova, F. D. (2016). IL-17A and multiple sclerosis: signaling pathways, producing cells and target cells in the central nervous system. Curr. Drug Targets 17, 1882-1893. doi: 10.2174/ 1389450117666160307144027

Komiyama, Y., Nakae, S., Matsuki, T., Nambu, A., Ishigame, H., Kakuta, S., et al. (2006). IL-17 plays an important role in the development of experimental autoimmune encephalomyelitis. J. Immunol. 177, 566-573. doi: 10.4049/ jimmunol.177.1.566

Kostic, M., Dzopalic, T., Zivanovic, S., Zivkovic, N., Cvetanovic, A., Stojanovic, I., et al. (2014). IL-17 and glutamate excitotoxicity in the pathogenesis of multiple sclerosis. Scand. J. Immunol. 79, 181-186. doi: 10.1111/sji.12147

Li, G. Z., Zhong, D., Yang, L. M., Sun, B., Zhong, Z. H., Yin, Y. H., et al. (2005). Expression of interleukin-17 in ischemic brain tissue. Scand. J. Immunol. 62, 481-486. doi: 10.1111/j.1365-3083.2005.01683.x

Li, Z., Li, K., Zhu, L., Kan, Q., Yan, Y., Kumar, P., et al. (2013). Inhibitory effect of IL-17 on neural stem cell proliferation and neural cell differentiation. BMC Immunol. 14:20. doi: 10.1186/1471-2172-14-20

Liu, H., Liu, J., Xu, E., Tu, G., Guo, M., Liang, S., et al. (2017a). Human immunodeficiency virus protein Tat induces oligodendrocyte injury by enhancing outward K(+) current conducted by KV1.3. Neurobiol. Dis. 97, 1-10. doi: 10.1016/j.nbd.2016.10.007

Liu, S. Q., Zhang, M. L., Zhang, H. J., Liu, F. Z., Chu, R. J., Zhang, G. X., et al. (2017b). Matrine promotes oligodendrocyte development in CNS autoimmunity through the PI3K/Akt signaling pathway. Life Sci. 180, 36-41. doi: 10.1016/j.lfs.2017.05.010

Liu, J., Xu, P., Collins, C., Liu, H., Zhang, J., Keblesh, J. P., et al. (2013). HIV-1 Tat protein increases microglial outward $\mathrm{K}(+)$ current and resultant neurotoxic activity. PLoS One 8:e64904. doi: 10.1371/journal.pone.0064904

Lock, C., Hermans, G., Pedotti, R., Brendolan, A., Schadt, E., Garren, H., et al. (2002). Gene-microarray analysis of multiple sclerosis lesions yields new targets validated in autoimmune encephalomyelitis. Nat. Med. 8, 500-508. doi: 10. 1038/nm0502-500

Lucchinetti, C., Bruck, W., Parisi, J., Scheithauer, B., Rodriguez, M., and Lassmann, H. (1999). A quantitative analysis of oligodendrocytes in multiple sclerosis lesions. a study of 113 cases. Brain 122(Pt 12), 2279-2295. doi: 10.1093/brain/ 122.12 .2279
Maezawa, I., Nguyen, H. M., Di Lucente, J., Jenkins, D. P., Singh, V., Hilt, S., et al. (2018). Kv1.3 inhibition as a potential microglia-targeted therapy for Alzheimer's disease: preclinical proof of concept. Brain 141, 596-612. doi: 10.1093/brain/awx346

Mi, S., Lee, X., Shao, Z., Thill, G., Ji, B., Relton, J., et al. (2004). LINGO-1 is a component of the Nogo-66 receptor/p75 signaling complex. Nat. Neurosci. 7, 221-228. doi: $10.1038 / \mathrm{nn} 1188$

Mi, S., Miller, R. H., Lee, X., Scott, M. L., Shulag-Morskaya, S., Shao, Z., et al. (2005). LINGO-1 negatively regulates myelination by oligodendrocytes. Nat. Neurosci. 8, 745-751. doi: $10.1038 / \mathrm{nn} 1460$

Milovanovic, J., Arsenijevic, A., Stojanovic, B., Kanjevac, T., Arsenijevic, D., Radosavljevic, G., et al. (2020). Interleukin-17 in chronic inflammatory neurological diseases. Front. Immunol. 11:947. doi: 10.3389/fimmu.2020.00947

Moser, T., Akgun, K., Proschmann, U., Sellner, J., and Ziemssen, T. (2020). The role of TH17 cells in multiple sclerosis: therapeutic implications. Autoimmun Rev. 19:102647. doi: 10.1016/j.autrev.2020.102647

Nasrabady, S. E., Rizvi, B., Goldman, J. E., and Brickman, A. M. (2018). White matter changes in Alzheimer's disease: a focus on myelin and oligodendrocytes. Acta Neuropathol. Commun. 6:22.

Oksala, N. K., Oksala, A., Pohjasvaara, T., Vataja, R., Kaste, M., Karhunen, P. J., et al. (2009). Age related white matter changes predict stroke death in long term follow-up. J. Neurol. Neurosurg. Psychiatry 80, 762-766. doi: 10.1136/jnnp. 2008.154104

Paintlia, M. K., Paintlia, A. S., Singh, A. K., and Singh, I. (2011). Synergistic activity of interleukin-17 and tumor necrosis factor-alpha enhances oxidative stress-mediated oligodendrocyte apoptosis. J. Neurochem. 116, 508-521. doi: 10.1111/j.1471-4159.2010.07136.x

Perez-Verdaguer, M., Capera, J., Serrano-Novillo, C., Estadella, I., Sastre, D., and Felipe, A. (2016). The voltage-gated potassium channel Kv1.3 is a promising multitherapeutic target against human pathologies. Expert Opin. Ther. Targets 20, 577-591. doi: 10.1517/14728222.2016.1112792

Prentis, P. J., Pavasovic, A., and Norton, R. S. (2018). Sea anemones: quiet achievers in the field of peptide toxins. Toxins (Basel) 10:36. doi: 10.3390/toxins10010036

Pruss, H., Dewes, M., Derst, C., Fernandez-Klett, F., Veh, R. W., and Priller, J. (2011). Potassium channel expression in adult murine neural progenitor cells. Neuroscience 180, 19-29. doi: 10.1016/j.neuroscience.2011.02.021

Pugliatti, M., Sotgiu, S., and Rosati, G. (2002). The worldwide prevalence of multiple sclerosis. Clin. Neurol. Neurosurg. 104, 182-191. doi: 10.1016/s03038467(02)00036-7

Rodgers, J. M., Robinson, A. P., Rosler, E. S., Lariosa-Willingham, K., Persons, R. E., Dugas, J. C., et al. (2015). IL-17A activates ERK1/2 and enhances differentiation of oligodendrocyte progenitor cells. Glia 63, 768-779. doi: 10.1002/glia. 22783

Rus, H., Pardo, C. A., Hu, L., Darrah, E., Cudrici, C., Niculescu, T., et al. (2005). The voltage-gated potassium channel Kv1.3 is highly expressed on inflammatory infiltrates in multiple sclerosis brain. Proc. Natl. Acad. Sci. U S A. 102, 1109411099. doi: $10.1073 /$ pnas. 0501770102

Schmitz, A., Sankaranarayanan, A., Azam, P., Schmidt-Lassen, K., Homerick, D., Hansel, W., et al. (2005). Design of PAP-1, a selective small molecule Kv1.3 blocker, for the suppression of effector memory $\mathrm{T}$ cells in autoimmune diseases. Mol. Pharmacol. 68, 1254-1270. doi: 10.1124/mol.105.015669

Shimizu, T., Kagawa, T., Wada, T., Muroyama, Y., Takada, S., and Ikenaka, K. (2005). Wnt signaling controls the timing of oligodendrocyte development in the spinal cord. Dev. Biol. 282, 397-410. doi: 10.1016/j.ydbio.2005.03.020

Smith, G. S., Homchaudhuri, L., Boggs, J. M., and Harauz, G. (2012). Classic 18.5- and 21.5-kDa myelin basic protein isoforms associate with cytoskeletal and SH3-domain proteins in the immortalized N19-oligodendroglial cell line stimulated by phorbol ester and IGF-1. Neurochem. Res. 37, 1277-1295. doi: 10.1007/s11064-011-0700-2

Sriram, S., and Steiner, I. (2005). Experimental allergic encephalomyelitis: a misleading model of multiple sclerosis. Ann. Neurol. 58, 939-945. doi: 10.1002/ ana. 20743

Tao, Y., Zhang, X., Chopra, M., Kim, M. J., Buch, K. R., Kong, D., et al. (2014). The role of endogenous IFN-beta in the regulation of Th17 responses in patients with relapsing-remitting multiple sclerosis. J. Immunol. 192, 5610-5617. doi: 10.4049/jimmunol.1302580

Tegla, C. A., Cudrici, C., Rozycka, M., Soloviova, K., Ito, T., Singh, A. K., et al. (2011). C5b-9-activated, K(v)1.3 channels mediate oligodendrocyte cell cycle 
activation and dedifferentiation. Exp. Mol. Pathol. 91, 335-345. doi: 10.1016/j. yexmp.2011.04.006

Trapp, B. D. (2004). Pathogenesis of multiple sclerosis: the eyes only see what the mind is prepared to comprehend. Ann. Neurol. 55, 455-457. doi: 10.1002/ana. 20087

Tsiperson, V., Gruber, R. C., Goldberg, M. F., Jordan, A., Weinger, J. G., Macian, F., et al. (2013). Suppression of inflammatory responses during myelin oligodendrocyte glycoprotein-induced experimental autoimmune encephalomyelitis is regulated by AKT3 signaling. J. Immunol. 190, 1528-1539. doi: 10.4049/jimmunol.1201387

Tzartos, J. S., Friese, M. A., Craner, M. J., Palace, J., Newcombe, J., Esiri, M. M. et al. (2008). Interleukin-17 production in central nervous system-infiltrating $\mathrm{T}$ cells and glial cells is associated with active disease in multiple sclerosis. Am. J. Pathol. 172, 146-155. doi: 10.2353/ajpath.2008.070690

Vassall, K. A., Bessonov, K., De Avila, M., Polverini, E., and Harauz, G. (2013). The effects of threonine phosphorylation on the stability and dynamics of the central molecular switch region of $18.5-\mathrm{kD}$ myelin basic protein. PLoS One 8:e68175. doi: 10.1371/journal.pone.0068175

Vautier, F., Belachew, S., Chittajallu, R., and Gallo, V. (2004). Shaker-type potassium channel subunits differentially control oligodendrocyte progenitor proliferation. Glia 48, 337-345. doi: 10.1002/glia.20088

Vicente, R., Escalada, A., Villalonga, N., Texido, L., Roura-Ferrer, M., MartinSatue, M., et al. (2006). Association of Kv1.5 and Kv1.3 contributes to the major voltage-dependent $\mathrm{K}+$ channel in macrophages. J. Biol. Chem. 281, 37675-37685.

Waisman, A., Hauptmann, J., and Regen, T. (2015). The role of IL-17 in CNS diseases. Acta Neuropathol. 129, 625-637. doi: 10.1007/s00401-015-1402-7

Wang, C., Zhang, C. J., Martin, B. N., Bulek, K., Kang, Z., Zhao, J., et al. (2017). IL17 induced NOTCH1 activation in oligodendrocyte progenitor cells enhances proliferation and inflammatory gene expression. Nat. Commun. 8:15508.

Wang, X., Li, G., Guo, J., Zhang, Z., Zhang, S., Zhu, Y., et al. (2019). Kv1.3 Channel as a key therapeutic target for neuroinflammatory diseases: state of the art and beyond. Front. Neurosci. 13:1393. doi: 10.3389/fnins.2019. 01393

Warsi, J., Fezai, M., Fores, M., Elvira, B., and Lang, F. (2015). Up-Regulation of Voltage Gated K+ channels Kv1.3 and Kv1.5 by protein kinase PKB/Akt. Cell Physiol. Biochem. 37, 2454-2463. doi: 10.1159/000438598

Wu, M., Xu, L., Wang, Y., Zhou, N., Zhen, F., Zhang, Y., et al. (2018). S100A8/A9 induces microglia activation and promotes the apoptosis of oligodendrocyte precursor cells by activating the NF-kappaB signaling pathway. Brain Res. Bull. 143, 234-245. doi: 10.1016/j.brainresbull.2018.09.014

Xie, D., Ge, X., Ma, Y., Tang, J., Wang, Y., Zhu, Y., et al. (2020). Clemastine improves hypomyelination in rats with hypoxic-ischemic brain injury by reducing microglia-derived IL-1 $\beta$ via P38 signaling pathway. J. Neuroinflamm. $17: 57$.

Yuan, X. L., Zhao, Y. P., Huang, J., Liu, J. C., Mao, W. Q., Yin, J., et al. (2018). A Kv1.3 channel-specific blocker alleviates neurological impairment through inhibiting T-cell activation in experimental autoimmune encephalomyelitis. CNS Neurosci. Ther. 24, 967-977. doi: 10.1111/cns.12848

Zhao, Y., Qiu, W., Liu, J., Yuan, X., Mao, W., Yin, J., et al. (2020). Blockade of Kv1.3 potassium channel inhibits CD8(+) T cell-mediated neuroinflammation via PD-1/Blimp-1 signaling. Faseb J. 34, 15492-15503. doi: 10.1096/fj.202000861rr

Conflict of Interest: The authors declare that the research was conducted in the absence of any commercial or financial relationships that could be construed as a potential conflict of interest.

Copyright (c) 2021 Liu, Yang, Yang, Yuan, Wang, Zhang, Xiong and Xu. This is an open-access article distributed under the terms of the Creative Commons Attribution License (CC BY). The use, distribution or reproduction in other forums is permitted, provided the original author(s) and the copyright owner(s) are credited and that the original publication in this journal is cited, in accordance with accepted academic practice. No use, distribution or reproduction is permitted which does not comply with these terms. 